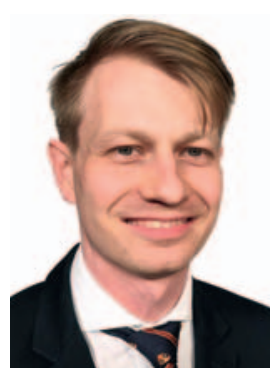

Sebastian Zimmer

Koordination «DermaCampus»

\section{Arztsein - lernen, lachen, Erfahrungen sammeln}

Herzlich willkommen zur dritten Ausgabe des «DermaCampus» in diesem Jahr! Auch dieses Mal haben wir wieder praxisrelevante Inhalte für Sie zusammengestellt, die für Ihre Facharztweiterbildung und das «Arztsein» generell nützlich sind.

Wer nicht eine Famulatur oder ein PJ-Tertial in der Dermatologie verbracht hat, steht am Beginn seiner Weiterbildung vor dem Mysterium der Immunfluoreszenz-Diagnostik. Oft besteht Uneinigkeit unter den Kolleginnen und Kollegen, wo die Hautprobe für die direkte Immunfluoreszenz überhaupt entnommen werden soll, da diese, je nach Verdachtsdiagnose, läsional oder aber periläsional zu entnehmen ist. Welches Medium sollte verwendet werden bzw. nicht verwendet werden? Was geschieht im Histologielabor mit der Hautprobe? Was ist der Unterschied zur indirekten Immunfluoreszenz? Diese Fragen möchte Dr. Florian Butsch mit Ihnen in seinem Beitrag in dieser Ausgabe des «DermaCampus» gerne klären.

Mindestens ein Funke Wahrheit liegt in dem Spruch «Lachen ist die beste Medizin». Dies weiß man nicht erst seit Comedians wie Dr. Eckhart von Hirschhausen. Zu Beginn der Karriere glaubt man vor allem an die Kraft der modernen Medizin, doch im Laufe des Berufslebens wird man auf die Wirkung der Kommunikation bzw. des Wortes aufmerksam. Dagegen allerdings tritt parallel der Verlust des Humors im Umgang mit Patienten und der Kollegenschaft ein. Den Humor nicht zu verlieren oder auch neu zu entflammen, lohnt sich nicht nur für die Arzt-Patienten-Beziehung, sondern auch für das ganz persönliche Wohl - denn: ein kranker Helfer ist ein schlechter Helfer. Dr. Christoph Krause zeigt uns, wie wichtig Humor im ärztlichen Berufsalltag ist.

Außerdem werden zwei Dermatologen, Roberto Souto aus Brasilien und Ricci Francesco aus Italien einen Einblick in das Berufsleben des Arztes in ihrem Heimatland geben. Es geht unter anderem um die Gründe für die Berufswahl, Restriktionen im Gesundheitssystem und die soziale Stellung des Arztberufs in der Gesellschaft.

Ich wünsche Ihnen nun eine anregende Lektüre! Mit herzlichen Grüßen,

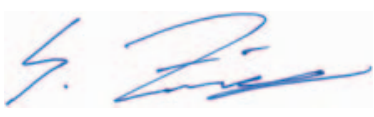

\section{KARGER}

Fax +497614520714 information@karger.com www.karger.com (c) 2018 S. Karger GmbH, Freiburg
Dr. Sebastian Zimmer 
Wichtige Diagnoseverfahren im Überblick Immunfluoreszenz-Diagnostik in der Dermatologie

Die Immunfluoreszenz gehört zusammen mit Immunhistochemie und Molekularpathologie zu den wichtigsten Ergänzungen der konventionellen Lichtmikroskopie in der Dermatohistologie. Besonderen Stellenwert hat sie für die Diagnostik bei bullösen Autoimmundermatosen, Kollagenosen und Vaskulitiden. Im Rahmen der Diagnostik bei Lichen planus und Porphyria cutanea tarda findet die Immunfluoreszenz ebenfalls Anwendung, hat hier jedoch eine weniger hervorgehobene Bedeutung [1]. Ihren Einzug in die Dermatohistologie hielt die Immunfluoreszenz Mitte des 20. Jahrhunderts durch die Beschreibung des «Lupusband-Tests» in der direkten Immunfluoreszenz und den Nachweis zirkulierender Autoantikörper im Serum an Pemphigus vulgaris leidender Patienten in der indirekten Immunfluoreszenz [2, 3].
Formalin befüllte Transportröhrchen gegeben werden, um Kontaminationen der DIF-Probe durch der Pinzette anhaftendes Formalin zu vermeiden. Die DIF weist gewebeständige Niederschläge von Fibrinogen, Komplement, IgG, IgM und IgA nach. Dazu wird ein Kryoschnitt der Biopsie mit Fluorochrom-gekoppelten DetektionsAntikörpern inkubiert, die für die nachzuweisenden Antikörper spezifisch sind. Anschließend können die aus Zielstruktur und Detektionsantikörper bestehenden Komplexe durch Fluoreszenz im Gewebe visualisiert werden [1, 2] (Abb. 1).

\section{Durchführung einer indirekten Immunfluoreszenz}

Die indirekte Immunfluoreszenz (IIF) dient dem Nachweis zirkulierender Autoantikörper im Serum des Patienten. Die IIF ist für die

\section{Das Prinzip der Immunfluoreszenz}

Das Prinzip der Immunfluoreszenz beruht auf der Eigenschaft der sogenannten Fluorochrome, spontan Licht einer bestimmten Wellenlänge zu emittieren, kurz nachdem sie durch ein im Vergleich energiereicheres und somit kurzwelligeres Licht ( $E=h^{*} f$ und $c=\lambda^{*} f=$ const.) angeregt wurden. Die in der Immunfluoreszenz-Diagnostik heute gebräuchlichen Fluorochrome sind Fluoresceinisothiocyanat (FITC), das grün fluoresziert und Tetramethylrhodamin-Isothyiocyanat (TRITC), das eine rote Fluoreszenz aufweist.

\section{Durchführung einer direkten \\ Immunfluoreszenz}

Für die direkte Immunfluoreszenz (DIF) wird eine Hautbiopsie benötigt. Bei bullösen Autoimmundermatosen sollte diese unmittelbar periläsional entnommen werden. Bei Kollagenosen und Vaskulitiden wird hingegen läsionale Haut für die DIF benötigt. Bei der Frage nach einer Vaskulitis sollte eine möglichst frische Läsion biopsiert werden, da sonst gewebeständige IgA-Antikörper-Niederschläge gegebenenfalls nicht mehr nachweisbar sind. Die Biopsie für die DIF darf nicht in Formalin fixiert werden und muss stattdessen in flüssigem Stickstoff oder «Michel-Medium» in das Labor transportiert werden. Auch kleinste Kontaminationen durch Formalin können die Probe unbrauchbar für die DIF machen [2]. Daher sollte immer zunächst die Probe für die DIF in das entsprechende Transportröhrchen eingebracht und erst dann die Probe für die Histologie in das mit

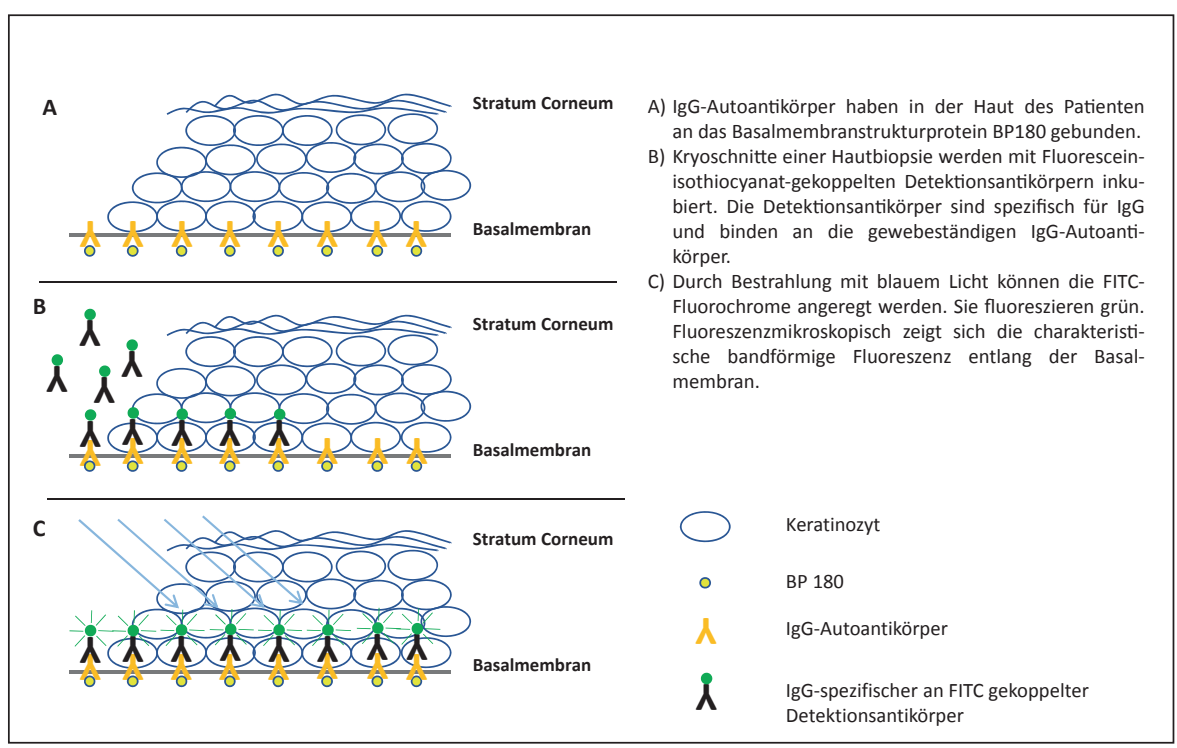

Abb. 1. Prinzip der direkten Immunfluoreszenz am Beispiel des Bullösen Pemphigoids @ Dr. Florian Butsch

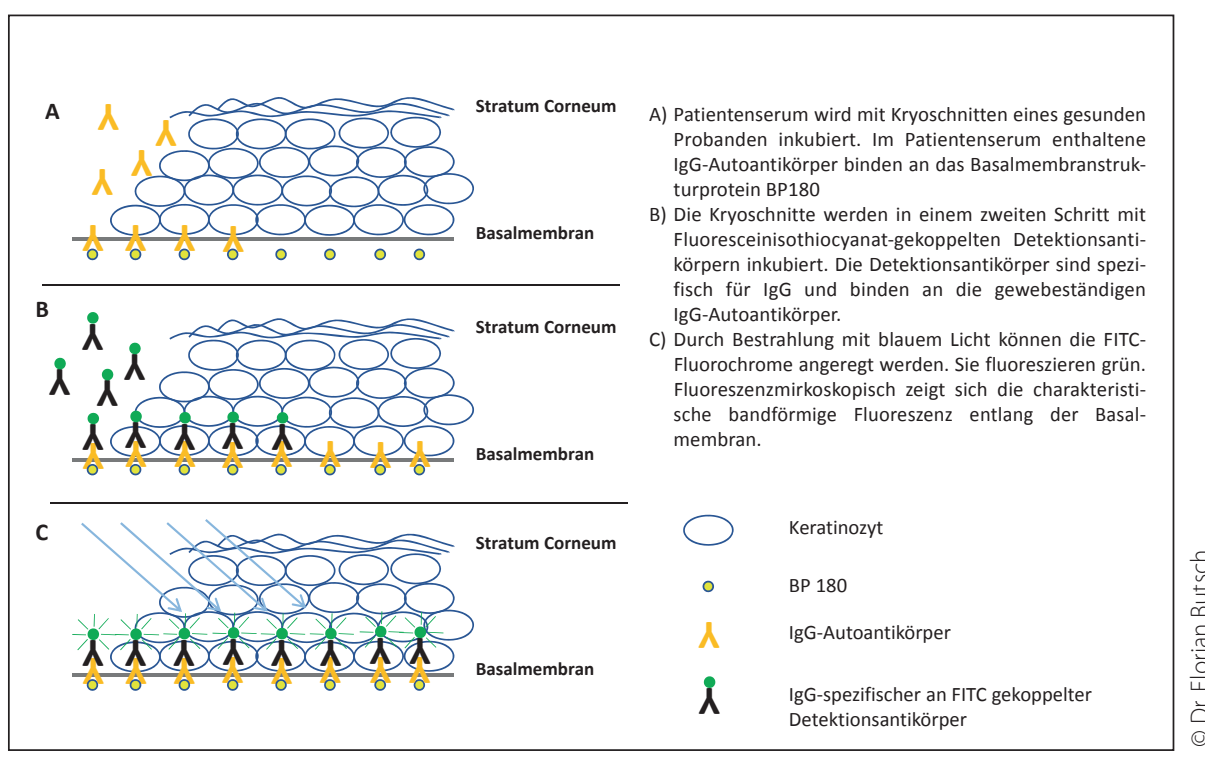

Abb. 2. Prinzip der indirekten Immunfluoreszenz an Normalhaut am Beispiel des Bullösen Pemphigoids 
Abb. 3. Befunde der Immunfluoreszenz bei blasenbildenden Autoimmundermatosen (c) Prof. Dr. Esther von Stebut-Borschitz
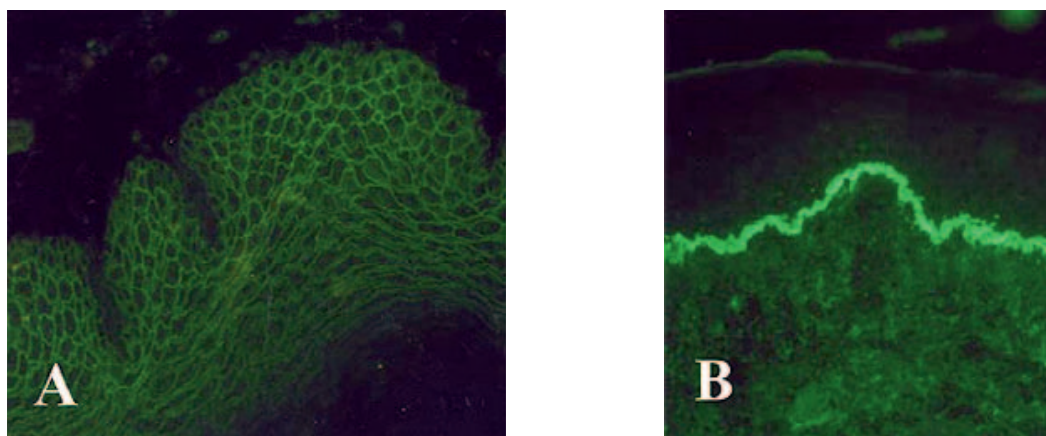

Befunde der Immunfluoreszenz bei blasenbildenden Autoimmundermatosen

A) Pemphigus vulgaris. Charakteristisch netzförmige Immunfluoreszenz durch Bindung der Autoantikörper an Strukturproteine der Desmosomen in den Interzellularräumen (ICS)

B) Bullöses Pemphigoid. Charakteristisch bandförmige Immunfluoreszenz durch Bindung der Autoantikörper an Strukturproteine der Basalmembran
Diagnostik bullöser Autoimmundermatosen von besonderer Bedeutung und hilft nicht nur bei der qualitativen Diagnose, sondern kann hier auch zur Evaluation der Krankheitsaktivität herangezogen werden [4,5]. Die Standardverfahren der IIF sind Untersuchungen an humaner Normalhaut und humaner $\mathrm{NaCl}$-Spalthaut. Je nach klinischer Fragestellung können ergänzende Untersuchungen an Epithelien des Affenösophagus und der Rattenblase erfolgen. Schnitte des ausgewählten Substrats werden mit verschiedenen Verdünnungen des Patientenserums inkubiert. Sofern Autoantikörper gegen Strukturen der Haut im Serum des Patienten enthalten sind, binden diese an die entsprechenden Zielantigene im Gewebe und können anschließend in einem zweiten Arbeitsschritt durch Fluorochrom-gekoppelte Detektions-Antikörper und Fluoreszenz-Untersuchung sichtbar gemacht werden. Es sollten stets mehrere Titer-Stufen des Serums untersucht werden. Dies erlaubt zum einen bei positiven Befunden eine Aussage über die Krankheitsaktivität - insbesondere wenn Verlaufsuntersuchungen durchgeführt werden, andererseits kann so bei negativen Befunden das Prozonen-Phänomen ausgeschlossen werden. Das Prozonen-Phänomen beschreibt falsch negative Befunde bei Verwendung schwach verdünnter Serum-Proben und sehr hoher Antikörper-Konzentration im Serum, was durch den Überschuss an Antikörpern letztlich zu einer Inhibition der Antigen-AntikörperBindung führt $[1,2,4]$.

In der jüngeren Vergangenheit hat die IIF durch die Verwendung von ELISA (enzyme-linked immunosorbent assay), Immunblot und Biochips nützliche Erweiterungen erfahren, welche die diagnostische Aussagekraft der IIF bei bullösen Autoimmundermatosen deutlich erweitert haben. Diese Methoden erlauben den Nachweis von Auto-Antikörpern gegenüber spezifischen Antigenen der Haut und ermöglichen so eine differenzierte Diagnostik. Verschiedene ready-to-use Biochips sind kommerziell verfügbar und erlauben die Diagnose von Pemphigus vulgaris, Pemphigus foliaceus, bullösem Pemphigoid und Epidermolysis bullosa aquisita mit hoher Sensitivität und Spezifität $[2,4,6]$ (Abb. 2).

\section{Fazit für die Praxis}

Zusammenfassend ist die Immunfluoreszenz ein wichtiger Baustein der Diagnostik bei bullösen Autoimmundermatosen, Kollagenosen und Vaskulitiden (Abb. 3). Das grundlegende Verständnis der DIF und IIF ist von Bedeutung für den sachgerechten Einsatz dieser Methoden im klinischen Alltag der Dermatologie.

\section{Literatur}

1 Kalaaji AN, Nicolas MEO. Mayo clinic atlas of immunofluorescence in dermatology: patterns and target antigens, ed 1. Boca Raton, 2006

2 Shetty VM, Subramaniam K, Rao R. Utility of immunofluorescence in dermatology. Indian Dermatol Online J 2017;8:1-8.

3 Mehta V, Sarda A, Balachandran C. Lupus band test. Indian J Dermatol Venereol Leprol 2010;76:298-300.

4 Schmidt E, Goebeler M, Hertl M, et al.: S2k guideline for the diagnosis of pemphigus vulgaris/foliaceus and bullous pemphigoid. J Dtsch Dermatol Ges 2015;13:713-727.

5 Otten JV, Hashimoto T, Hertl M, et al.: Molecular diagnosis in autoimmune skin blistering conditions. Curr Mol Med 2014;14:69-95.

6 Van Beek N, Rentzsch K, Probst C, et al.: Serological diagnosis of autoimmune bullous skin diseases: prospective comparison of the BIOCHIP mosaic-based indirect immunofluorescence technique with the conventional multi-step single test strategy. Orphanet J Rare Dis 2012;7:49.

Kontaktadresse: Dr. Florian Butsch, Universitätsmedizin der Johannes Gutenberg-Universität Mainz, Haut- und Poliklinik, Geb. 401, CRC, Langenbeckstraße 1, 55131 Mainz, Deutschland, Florian.Butsch@unimedizinmainz.de. 
Arzt-Patienten-Kommunikation

Ein Appell für mehr Humor im årztlichen Berufsalltag!

Humor ist in aller Munde: bei Jan Böhmermann oder der Serie «Die Anstalt», bei Otto Waalkes oder Loriot, bei Dr. Eckart von Hirschhausen oder Vince Ebert. Aber: gibt es auch kompetente und humorvolle praktizierende Ärztinnen und Ärzte? Denn ein Blick in den ärztlichen Berufsalltag legt nahe, dass der Humor proportional zum Aufstieg auf der Karriereleiter häufig abnimmt.

Die Kombination aus humorvoll und kompetent kennt meist nur ein Kollege, der eine Kollegin hatte, die einen Kollegen kennt, der so ist. Aber selbst erlebt, hat das noch niemand. Nur bei sich selbst natürlich - denn sind wir nicht alle unglaublich feinfühlig und haben bestechenden Witz?

Wenn wir uns jetzt unter den Kollegen umschauen, gibt es ein paar, mit denen können wir lachen. Ein Großteil taugt zur professionellen Zusammenarbeit und der Rest der Gaußschen Verteilung ist gelinde gesagt eine Zumutung. Aber alle glauben bzw. sind überzeugt davon, in Sachen Kommunikation und Humor etwas drauf zu haben. Woran liegt das? Berufs- und Lebenserfahrung? Aus- und Fortbildungen in ärztlicher Kommunikation? Oder weil wir von Kindesbeinen an sprechen gelernt haben?

«Humor ist der Knopf, der verhindert, dass uns der Kragen platzt.»

Joachim Ringelnatz

Wenn wir ehrlich sind, haben die meisten von uns einen Strahlenschutz-Kurs, einen Abdomen-Ultraschall-Kurs oder einen BLSKurs besucht. Aber gilt das auch für einen Kurs in ärztlicher Kommunikation oder noch spezieller zum Thema «Humor in der Medizin»? Fehlanzeige! Dabei führen Ärztinnen und Ärzte gut 200000 Gespräche im Laufe ihrer Karriere. In einem Bereich der hochsensibel für Missverständnisse, Kompetenzgerangel und anstrengende Patienten ist. Nicht schlimm, das kann man ändern. Die Initiative «Arzt mit Humor» hat sich auf die Fahnen geschrieben, den Humor auch im Krankenhaus oder der Arztpraxis salonfähig zu machen. Denn die Gaußsche Verteilung der Kolleginnen und Kollegen mit Humor entsteht nicht, weil das Gegenüber keinen hat, sondern weil es Ihren Humor nicht teilt oder eben einen anderen hat. Dasselbe gilt übrigens auch für Patienten, ihre Angehörigen und das Pflegepersonal.

Wir wundern uns manchmal, wieviel «Arzt» ein Patient verträgt. Weg von der rein medizinischen Sichtweise interessiert mich, wieviel Humor haben meine Patienten, meine Kollegen, das Pflegepersonal noch bzw. wieviel geht noch? Oder warum ist der Humor abhandengekommen? Oder kann Humor meinem Problem schaden? Man weiß, dass sich in entspannten Situationen besser lernen lässt, dass Humor die Aufmerksamkeit erhöht und er im medizinischen
Kontext u.a. die kardiopulmonale Physiologie positiv beeinflusst, dass er ablenken kann, die Perspektive ändert und damit Ängste reduziert.

«Kommen eine Seele und ein Körper gemeinsam ins Krankenhaus. Sagt die Seele zum Körper: «Geh ruhig vor. Dich verstehen sie hier besser.»»

Ulrich Schaffer

Im medizinischen Kontext fehlt es nicht an Humor. Er bedient nur zu schnell Schenkelklopfer, wird zu plump oder grenzt unsere PeerGroups ab. Chirurgen und Anästhesisten können davon ein Lied singen. «Wie nennt man das grüne Tuch zwischen OP-Feld und Beatmung? Blut-Hirn-Schranke!» Das macht zwar kurzfristig Spaß, verbessert langfristig aber die Zusammenarbeit nicht.

Feinsinnig seinen humorvollen Fingerabdruck im stressigen Berufsalltag zu hinterlassen, das ist eine ärztliche Kunst, die begeistert. Mein Gegenüber humorvoll aufzuwerten, ein Ventil für Druck und Stress zu haben und sich selbst vor dem Ausbrennen zu bewahren, das alles kann ein spezielles Training bei «Arzt mit Humor» bewirken. Denn den Humor bringen Sie mit. Sie müssen inn nur bewusst nutzen und die Situation nicht dem Zufall überlassen. Nicht aufgesetzt und künstlich, sondern authentisch und echt. Das sind dann Sie! Wenn die eigene Leistungsfähigkeit steigt, die Freude im Arztberuf wiederkommt und Humor zur Erhaltung der eigenen Gesundheit oder im Konflikt eine wirksame Ressource wird, dann haben Sie wohl ein Training von «Arzt mit Humor» besucht und es hat Sie überzeugt.

Das nächste Training «Humor auf Rezept? Die Dosis bringt den Erfolg!» für Ärztinnen und Ärzte findet am 15. \& 16.03.2019 in Leipzig statt. Anmeldung und weitere Informationen unter www.arztmithumor.de.

Dr. Christoph Krause ist Anästhesist am Universitätsklinikum Leipzig und fährt als Notarzt durch die Stadt. Vor Jahren hat er zusammen mit dem «Deutschen Institut für Humor» die Initiative «Arzt mit Humor» ins Leben gerufen, weil der Berufsalltag nicht immer die wahre Freude war, wenn es um den Umgang zwischen der Ärzteschaft, den Studierenden, dem Pflegepersonal, den Patienten und den Angehörigen ging. Wohl wissend, dass es im Gesundheitswesen Faktoren gibt, die den Idealismus verschrecken, hält er an der Vision fest, gute ärztliche Kommunikation und Hochleistungsmedizin miteinander zu verbinden.

Kontaktadresse: Dr. Christoph Krause, Deutsches Institut für Humor (DIH) ${ }^{\circledR}$, Feuerbachstraße 26, 04105 Leipzig, Deutschland, christoph.krause@ arztmithumor.de. 


\section{Medizin international}

Being a doctor - perspectives from all around the world / Arztsein - Perspektiven von der ganzen Welt

Wie es deutschen Ärztinnen und Ärzten im Ausland geht, welche kulturellen Hürden sie überwinden und was sie lernen können, wissen wir aus zahlreichen Berichten. Auch die Sicht ausländischer Fachleute, die in Deutschland praktizieren ist ein interessanter Spiegel der hiesigen Verhältnisse. Weitestgehend unbekannt ist jedoch die Innenperspektive einheimischer Medizinerinnen und Mediziner in den verschiedenen Ländern der Welt, die weit mehr abbilden können als den medizinischen Kontext. Sie können den Finger unvermittelter, radikaler in die Wunde legen, ohne dem Impuls nachzugeben, sie gleich zu versorgen. Erst kürzlich hat der dramatische Hungerstreik vieler junger polnischer Ärzte diese Perspektive eröffnet, die mehr zutage gefördert hat als nur ein Überstundenproblem.

Wie lebt und arbeitet es sich als Ärztin und Arzt im Ausland? Was sagen die Verhältnisse über das soziale System und den Stellenwert des Menschen in der Gesellschaft aus?

Im DermaCampus stellen zwei Ärzte der Dermatologie aus Italien und Brasilien den Hintergrund ihrer Berufswahl und ihre Perspektiven auf Medizin und Gesellschaft in ihrem Land vor.

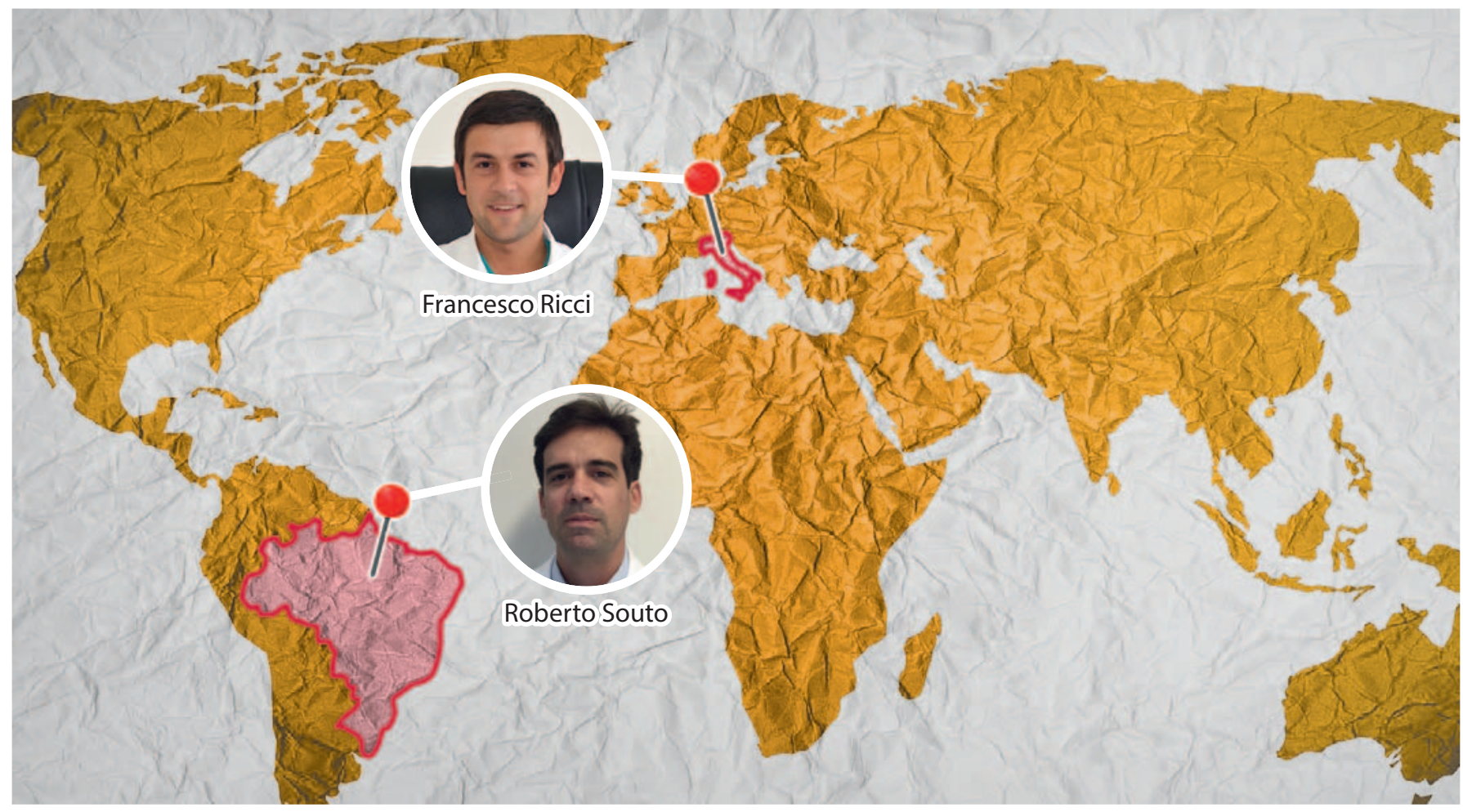

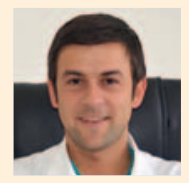

Francesco Ricci, PhD, MD; Head of Melanoma Unitinthelstituto Dermopatico dell'Immacolata di Roma, Italy (IDI-IRCCS, FLMM)
Why did you choose to become a doctor?

Francesco Ricci, Italy I decided to become a doctor because I think that being able to help people in solving health problems is one of the greatest gratifications of a person's life. Furthermore, I believe that the study of the human body and the related diseases is one of the most interesting issues.

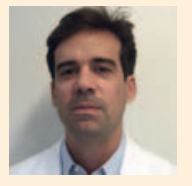

Roberto Souto, MD; Professor at Serviço Dermatologia Hospital Universitário Pedro Ernesto, Rio de Janeiro, Brazil
Roberto Souto, Brazil That was an option of a career that let me choose my own schedule. I have time to deal with a subject I like (dermatology) and spend enough time with who I love most (my family). 
Francesco Ricci, Italy I believe that the doctor's work will never be perfect because not all human diseases heal completely; some of them are simply best managed with the progress that the science offers us right now. Therefore sometimes we need to make it clear to the patient that the medicine is not an exact science.

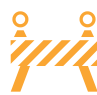

What are the main obstacles in your daily work?

Francesco Ricci, Italy Right now in Italy it is very difficult for young doctors to work in the public health care system because there are a few new recruitments and the contracts are often temporary. Many Italian young doctors can work in private practice only or they have to migrate abroad to find a stable job in the public health system of other countries.
Roberto Souto, Brazil In a perfect world we would have enough time for the patients, solve their medical problems without concern about treatment costs and exams, and have a good remuneration to study and be more able to solve others patients' diseases.
Roberto Souto, Brazil The health care system restricts our daily work. It's a reality that I believe almost all countries have. It is necessary to strike a balance between patient disease, treatment and exam costs, doctor remuneration, and prevention. This I believe could be the most important part of the puzzle: prevention.

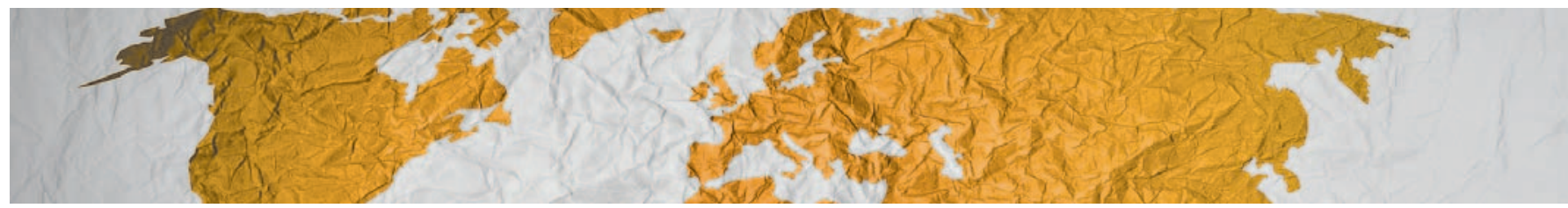

(1) How would you rate your job as a doctor in terms of acknowledgement in your society, compared to other jobs?

Francesco Ricci, Italy I think that a few years ago the doctor's role was recognized at a higher level than today. However, compared to other professions, I think that the doctor's job is still an occupation with a good rate of acknowledgement in our society.
Roberto Souto, Brazil I believe other professionals in other areas do not know how hard we work and study to bring the best to our patient. I would rate it at top 5.

\section{Would you make the same choice if you could choose again and why?}

Francesco Ricci, Italy Yes, I would make the same choice again. Even if it is a profession with a strong emotional involvement which requires a lot of time to devote to patients, the effort is rewarded with personal satisfaction.
Roberto Souto, Brazil Yes, for sure. No doubt. 